\title{
Symptomatic, frail, elderly patients: the urgent need for comprehensive assessment and management
}

\author{
Enrico Clini', Bianca Beghe ${ }^{2}$ and Leonardo M. Fabbri
}

\begin{abstract}
Affiliations: ${ }^{1}$ Villa Pineta di Gaiato, University of Modena and Reggio Emilia, Pavullo nel Frignano Modena, Italy. ${ }^{2}$ Department of Oncology, Hematology and Respiratory Diseases, Polyclinic of Modena, University of Modena and Reggio Emilia, Modena, Italy.
\end{abstract}

Correspondence: Leonardo M. Fabbri, Department of Oncology Hematology and Respiratory Diseases, Polyclinic of Modena, University of Modena and Reggio Emilia, Largo del Pozzo 71, 41124 Modena, Italy. E-mail: leonardo.fabbridunimore.it

@ERSpublications

Early screening of chronic diseases and COPD needs attention in the elderly population to properly assess management http://ow.ly/ACbi3

The working hypotheses for screening and early diagnosis of chronic obstructive pulmonary disease (COPD) have been that: 1) long-term exposure to cigarette smoke or pollutants damages the airways and/or the lungs, and causes airflow obstruction $[1,2] ; 2$ ) airflow obstruction occurs primarily in the small airways, is asymptomatic in the early stages of the disease and is progressive in the majority of COPD patients [1-4]; and 3) forced expiratory volume in $1 \mathrm{~s}$ (FEV1) and its ratio with forced vital capacity are necessary in establishing the diagnosis of COPD, and in assessing the excessive decline in lung function [1], but are of little use, if any, in making the early, pre-symptomatic diagnosis of COPD [5].

These working hypotheses have been helpful in stimulating research in the field of structure-function relationships in the lung, and particularly in defining the role of airway and lung inflammation in the development of central and peripheral airway remodelling and emphysema $[2,3,6]$. Research focused on the respiratory system has also brought about the development of physiological and imaging methods to assess peripheral airways and lung parenchyma [7-9], and has furthered understanding of the natural history of COPD, particularly the progressive decline in lung function and its modulation by pharmacological intervention [10]. Thanks to the results of these studies, we now know much more about COPD, about the potential and limitations of screening [11] and early diagnosis based on spirometry, and about interventions in early asymptomatic and newly discovered COPD.

Screening subjects at risk, such as smokers, workers and populations exposed to environmental pollution, with serial measurements of FEV1 has been shown to identify COPD at an early stage, with a diagnostic yield that is significant even in asymptomatic individuals [12]. Studies including measurement of small airway function, though inconclusive, have been used in an attempt to identify more subtle changes in airway function, without success [5, 13]. Novel imaging methods [8, 9] and biomarker profiles [14, 15] show promise, but we are not there yet. Consequently, a suitable alternative to spirometry has yet to be discovered. Spirometry is simple, inexpensive, and remains the best practical method for use in early detection and screening of undiagnosed airflow obstruction [16].

The rationale for identifying subjects with previously undiagnosed COPD and for early diagnosis of COPD in subjects at risk is that it would benefit the patient, either because symptoms and quality of life would be improved, exacerbations and complications would be limited, and survival would be enhanced, or because

Received: Aug 182014 | Accepted: Aug 192014

Conflict of interest: None declared.

Copyright (C)ER 2014 
the development and progression of the disease would be prevented by intervention (e.g. smoking cessation) or pharmacological treatment.

However, based on the evidence available, screening for COPD with spirometry is currently recommended only to establish the diagnosis of COPD in subjects over the age of 40 years who complain of chronic respiratory symptoms, are at risk (smokers and/or exposed to indoor outdoor pollutants) and have a family history of COPD [1]. By contrast, spirometry is not recommended for screening asymptomatic individuals [17], nor for screening adults at risk of COPD [18]. This is mainly because there is no evidence that COPD newly diagnosed by spirometry can be effectively treated or its progression prevented. There is no published study showing that undiagnosed COPD at any stage that is identified by screening benefits from intervention. In addition, there is no evidence that spirometry screening improves smoking cessation and no firm evidence that pharmacological interventions (e.g. with bronchodilators and/or inhaled steroids) reduce the progression of COPD, as assessed by both excess decline in lung function and mortality [19]. Even the role of COPD screening for lung cancer surveillance is still unclear [20].

All the aforementioned studies, however, focused mainly on COPD as a pulmonary disease and not as a pulmonary component of a complex chronic disease, as it is regarded today, particularly in the elderly $[1,21,22]$.

In an interesting study published in this issue of the European Respiratory Journal, BERTENS et al. [23] investigated a different aspect of COPD screening: whether screening for COPD in frail, elderly subjects complaining of dyspnoea is effective in discovering new cases of COPD, and whether it has an impact on patient management and prognosis. The simple answers are "yes" to the former and "no" to the latter.

This approach is novel in several respects. First, it focuses on a specific group of subjects at high risk of COPD: those who are symptomatic (dyspnoea is the most characteristic symptom of COPD), elderly (together with smoking, ageing is the main risk factor for COPD) and frail (subjects have $\geqslant 3$ diseases and receive $\geqslant 5$ drugs). Second, screening was based not only on spirometry but also on a detailed individual assessment by a multidisciplinary panel (a general practitioner, a cardiologist and a pulmonologist) that took into account clinical history, risk factors, physical examination, blood tests, ECG, echocardiography, and pre- and post-bronchodilator spirometry. Third, the screening of this frail, elderly population complaining of dyspnoea included careful assessment of heart failure (the other main cause of dyspnoea) and other causes of frequent dyspnoea in the elderly [24].

The study by BERTENS et al. [23] is a small part of a wider programme primarily aimed at determining the relationship between COPD and heart failure. These are the most common chronic diseases responsible for dyspnoea $[25,26]$. They share important risk factors (ageing and smoking), often occur together, and both are often underdiagnosed and undertreated, particularly in the elderly $[25,26]$. Patients with an established diagnosis of both COPD and heart failure were excluded from the study, whereas subjects with only one of the diagnoses were included. Interestingly, in the entire population of frail individuals, including those with both COPD and heart failure, the overall prevalence of COPD was almost $45 \%$ and the overall prevalence of heart failure was $>40 \%$. Taken together with the high prevalence of other chronic conditions (reported in table 1 of the article [23]), these data suggest a high prevalence of multimorbidity in this elderly and frail group of patients who complain of dyspnoea.

With this meticulous approach, the authors found that screening indeed identified a significant number of new cases of COPD, but also that these newly diagnosed subjects, although at higher risk of hospitalisation and exacerbations or pneumonia, had no significant change in their management in the following 6-12 months. The authors concluded that screening programmes for COPD in frail patients complaining of dyspnoea should therefore include the impact on long-term management and prognosis.

Although the study was relatively small, descriptive, with a short follow-up period (6-12 months) and was limited to reporting data on the screening for COPD, it emphasises both the effectiveness and the limitations of actively searching for COPD in this population. Even if in the present paper the authors report data only on COPD, in the main study, they indeed address COPD as the pulmonary component of multimorbidity. In fact, they use a meticulous approach to the possible causes of dyspnoea (heart failure, arrhythmia, ischaemic heart disease, anaemia, obesity, thyroid disease, etc.) and to targeted interventions in newly diagnosed disease [25].

The study raises several questions. 1) Why screen for COPD if there is no change in COPD management by the general practitioner? 2) Why screen only for COPD in frail, elderly subjects who may have other conditions or concomitant chronic diseases that contribute to dyspnoea? 3) Why screen for COPD and other chronic diseases without a concomitant strategy for treating COPD and concomitant chronic diseases? 
FIGURE 1 The management of the complex elderly patient with chronic obstructive pulmonary disease (COPD): moving from the single disease model to the needs in the complex patient.

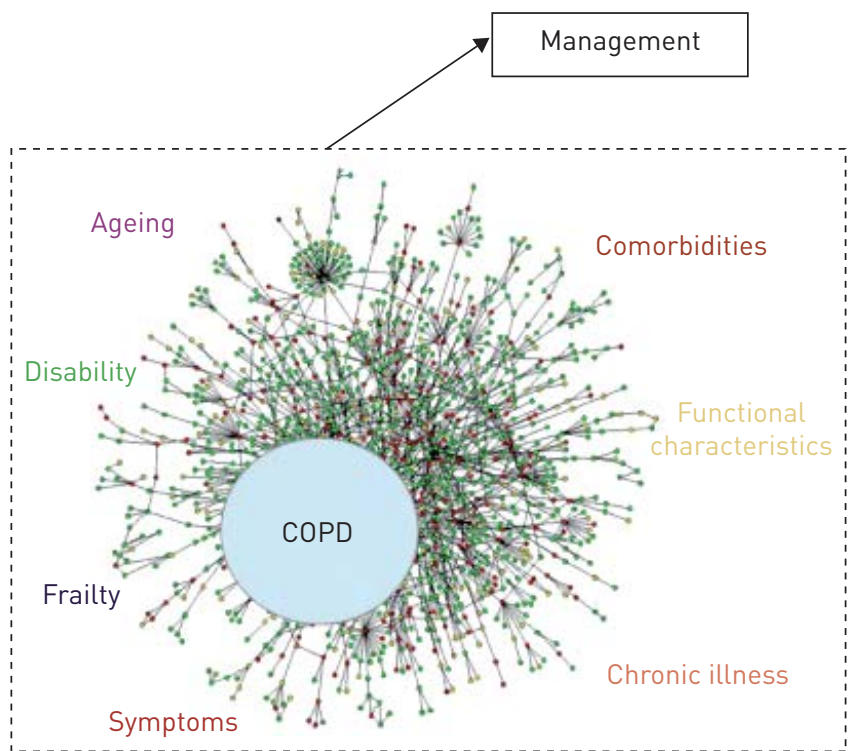

Screening for COPD is not associated with a change in management by the general practitioner for reasons that are well described by the authors. An additional reason may be that, unlike cardiovascular and metabolic diseases, there has been no clinical trial in this population of symptomatic, frail, elderly individuals, and thus, there is no evidence that any intervention proven to be effective in younger individuals with less severe disease is still effective in the elderly. This reinforces the urgent need for studies of this difficult population [26] and of patients with complex concomitant chronic diseases such as COPD and chronic heart failure [27].

Screening only for COPD in a frail, elderly population with various conditions or concomitant chronic diseases that may contribute to dyspnoea is obviously wrong. Although the authors recommend spirometry for screening COPD in symptomatic, frail, elderly individuals complaining of dyspnoea, the frailty and multimorbidity of these patients indicate that global conditions and metric characteristics, and concomitant chronic diseases should be properly searched for and taken into account for the test of an individual's performance and interventions [28]. Thus, it is unclear why the authors decided to report only data on COPD. The first key objective of their general study [25] was to determine the yield of screening for previously unrecognised COPD, heart failure, and other chronic diseases such as anaemia, thyroid disorders and renal dysfunction. All these conditions are also likely to be underdiagnosed and undertreated, and thus screening is likely to reveal them. It would be interesting to know the results of such a comprehensive screen and its consequences.

Why screen for COPD and other chronic conditions without implementing a concomitant strategy for treating these conditions? Indeed, screening and following a symptomatic, elderly COPD patient should not be limited simply to predicting the prognosis but should include intervention. Again, one of the key objectives of the overall study [25] was to assess the cost-effectiveness of diagnostic screening and of subsequent targeted interventions; thus, the results of their larger study are awaited so we can learn the answers to these questions.

The bottom line of the article by BERTENS et al. [23] and its conclusions is that we urgently need properly designed (size and duration of follow-up) and powered, controlled, randomised clinical trials to assess both the complexity of frail elderly patients complaining of dyspnoea and the cost-effectiveness of comprehensive interventions. These trials are very difficult to design but, for chronic diseases in general and for frail, elderly patients in particular, we urge our fellow investigators to move out of the single-disease model and to address the complexity of these patients and their pharmacological and nonpharmacological management (fig. 1) $[26,29,30]$

\section{References}

1 Vestbo J, Hurd SS, Agustí AG, et al. Global strategy for the diagnosis, management, and prevention of chronic obstructive pulmonary disease: GOLD executive summary. Am J Respir Crit Care Med 2013; 187: 347-365.

2 de Jong K, Boezen HM, Kromhout $\mathrm{H}$, et al. Occupational exposure to vapors, gases, dusts, and fumes is associated with small airways obstruction. Am J Respir Crit Care Med 2014; 189: 487-490.

3 McDonough JE, Yuan R, Suzuki M, et al. Small-airway obstruction and emphysema in chronic obstructive pulmonary disease. N Engl J Med 2011; 365: 1567-1575. 
Sanchez-Salcedo P, Divo M, Casanova, et al. Disease progression in young patients with COPD: rethinking the Fletcher and Peto model. Eur Respir J 2014; 44: 324-331.

5 van den Berge M, ten Hacken NH, Cohen J, et al. Small airway disease in asthma and COPD: clinical implications. Chest 2011; 139: 412-423.

6 Hogg JC. Pathophysiology of airflow limitation in chronic obstructive pulmonary disease. Lancet 2004; 364: 709-721. 7 van der Wiel E, ten Hacken NH, Postma DS, et al. Small-airways dysfunction associates with respiratory symptoms and clinical features of asthma: a systematic review. J Allergy Clin Immunol 2013; 131: 646-657.

8 Coxson HO, Leipsic J, Parraga G, et al. Using pulmonary imaging to move chronic obstructive pulmonary disease beyond FEV1. Am J Respir Crit Care Med 2014; 190: 135-144.

9 Camiciottoli G, Bigazzi F, Paoletti M, et al. Pulmonary function and sputum characteristics predict computed tomography phenotype and severity of COPD. Eur Respir J 2013; 42: 626-635.

10 Elbehairy AF, Webb KA, Neder JA, et al. Should mild COPD be treated? Evidence for early pharmacological intervention. Drugs 2013; 73: 1991-2001.

11 Celli BR. Counterpoint: should storefront clinics provide case finding and chronic care for COPD? No. Chest 2014; 145: 1193-1194.

12 Parr DG. Patient phenotyping and early disease detection in chronic obstructive pulmonary disease. Proc Am Thorac Soc 2011; 8: 338-349.

13 Gennimata SA, Palamidas A, Karakontaki F, et al. Pathophysiology of evolution of small airways disease to overt COPD. COPD 2010; 7: 269-275.

14 Agusti A, Sin DD. Biomarkers in COPD. Clin Chest Med 2014; 35: 131-141.

15 Casanova C, Celli BR. Microalbuminuria as a potential novel cardiovascular biomarker in patients with COPD. Eur Respir J 2014; 43: 951-953.

16 Maio S, Sherrill DL, MacNee W, et al. The European Respiratory Society spirometry tent: a unique form of screening for airway obstruction. Eur Respir J 2012; 39: 1458-1467.

17 Qaseem A, Wilt TJ, Weinberger SE, et al. Diagnosis and management of stable chronic obstructive pulmonary disease: a clinical practice guideline update from the American College of Physicians, American College of Chest Physicians, American Thoracic Society, and European Respiratory Society. Ann Intern Med 2011; 155: 179-191.

18 Lin K, Watkins B, Johnson T, et al. Screening for chronic obstructive pulmonary disease using spirometry: summary of the evidence for the U.S. Preventive Services Task Force. Ann Intern Med 2008; 148: 535-543.

19 Zuwallack RL, Nici L. Modifying the course of chronic obstructive pulmonary disease: looking beyond the FEV1. COPD 2012; 9: 637-648.

20 Sekine Y, Katsura H, Koh E, et al. Early detection of COPD is important for lung cancer surveillance. Eur Respir J 2012; 39: 1230-1240.

21 Faner R, Cruz T, López-Giraldo A, et al. Network medicine, multimorbidity and the lung in the elderly. Eur Respir J 2014; 44: 775-788.

22 Divo MJ, Carlos H, Martinez CH, et al. Ageing and the Epidemiology of multimorbidity. Eur Respir J 2014 ; in press.

23 Bertens LCM, Reitsma JB, van Mourik Y, et al. COPD detected with screening: impact on patient management and prognosis. Eur Respir J 2014; 44: 1571-1578.

24 van Mourik Y, Rutten FH, Moons KG, et al. Prevalence and underlying causes of dyspnoea in older people: a systematic review. Age Ageing 2014; 43: 319-326.

25 van Mourik Y, Moons KG, Bertens LC, et al. Triage of frail elderly with reduced exercise tolerance in primary care (TREE). A clustered randomized diagnostic study. BMC Public Health 2012; 12: 385.

26 Tannenbaum C. How to treat the frail elderly: The challenge of multimorbidity and polypharmacy. Can Urol Assoc J 2013; 7: Suppl. 4, S183-S185.

27 Vestbo J, Anderson J, Brook RD, et al. The Study to Understand Mortality and Morbidity in COPD (SUMMIT) study protocol. Eur Respir J 2013; 41: 1017-1022.

28 Pistelli R, Ferrara L, Misuraca C, et al. Practical management problems of stable chronic obstructive pulmonary disease in the elderly. Curr Opin Pulm Med 2011; 17: Suppl. 1, S43-S48.

29 Fabbri LM, Boyd C, Boschetto P, et al. How to integrate multiple comorbidities in guideline development: article 10 in Integrating and coordinating efforts in COPD guideline development. An official ATS/ERS workshop report. Proc Am Thorac Soc 2012; 9: 274-281.

30 FitzGerald JM, Poureslami I. The need for humanomics in the era of genomics and the challenge of chronic disease management. Chest 2014; 146: 10-12. 